S. Kouachi (Khenchela and Annaba)

\title{
EIGENVALUES AND EIGENVECTORS OF SOME TRIDIAGONAL MATRICES WITH NON-CONSTANT DIAGONAL ENTRIES
}

Abstract. We give explicit expressions for the eigenvalues and eigenvectors of some tridiagonal matrices with non-constant diagonal entries. Our techniques are based on the theory of recurrent sequences.

1. Introduction. We consider tridiagonal matrices of the form

$$
A_{n}=\left(\begin{array}{cccccc}
-\alpha+b_{1} & c_{1} & 0 & 0 & \ldots & 0 \\
a_{1} & b_{2} & c_{2} & 0 & \ldots & 0 \\
0 & a_{2} & b_{3} & \ddots & \ddots & \vdots \\
0 & 0 & \ddots & \ddots & \ddots & 0 \\
\vdots & \vdots & \ddots & \ddots & \ddots & c_{n-1} \\
0 & \ldots & \ldots & 0 & a_{n-1} & -\beta+b_{n}
\end{array}\right),
$$

where $a_{j}$ and $c_{j}, j=1, \ldots, n-1$, and $\alpha$ and $\beta$ are complex numbers. We suppose that

$$
a_{j} c_{j}=d^{2}, \quad j=1, \ldots, n-1,
$$

and

$$
b_{j}=\left\{\begin{array}{ll}
b_{1} & \text { if } j \text { is odd, } \\
b_{2} & \text { if } j \text { is even, }
\end{array} \quad j=1, \ldots, n-1,\right.
$$

where $d, b_{1}$ and $b_{2}$ are complex numbers with $d \neq 0$.

If $\sigma$ is a mapping (not necessarily a permutation) from the set of the integers from 1 to $n-1$ into the set $\mathbb{N}^{*}$ of integers different from zero, we

2000 Mathematics Subject Classification: Primary 15A18.

Key words and phrases: eigenvectors, tridiagonal matrices.

The author was supported by the National Agency of Development and Scientific Research (ANDRU) under grant 01/06/163/99. 
denote by $A_{n}(\sigma)$ the $n \times n$ matrix

$$
A_{n}(\sigma)=\left(\begin{array}{cccccc}
-\alpha+b_{1} & c_{\sigma_{1}} & 0 & 0 & \ldots & 0 \\
a_{\sigma_{1}} & b_{2} & c_{\sigma_{2}} & 0 & \ldots & 0 \\
0 & a_{\sigma_{2}} & b_{3} & \ddots & \ddots & \vdots \\
0 & 0 & \ddots & \ddots & \ddots & 0 \\
\vdots & \vdots & \ddots & \ddots & \ddots & c_{\sigma_{n-1}} \\
0 & \ldots & \ldots & 0 & a_{\sigma_{n-1}} & -\beta+b_{n}
\end{array}\right)
$$

and by $\Delta_{n}(\sigma)=\left|A_{n}(\sigma)-\lambda I_{n}\right|$ its characteristic polynomial. If $\sigma=$ id, where id is the identity, then $A_{n}(\mathrm{id})$ and its characteristic polynomial $\Delta_{n}(\mathrm{id})$ coincide with $A_{n}$ and $\Delta_{n}$ respectively. Our aim is to establish the eigenvalues and the corresponding eigenvectors of the matrices $A_{n}(\sigma)$.

We mention that the matrices $A_{n}(\sigma)$ are of circulant type when $\alpha=\beta=$ $a_{1}=a_{2}=\cdots=0$ and all the entries on the subdiagonal are equal. They are of Toeplitz type when $\alpha=\beta=0$ and all the entries on the subdiagonal are equal and those on the superdiagonal are also equal (see U. Grenander and G. Szegö [4]).

When $a_{1}=a_{2}=\cdots=c_{1}=c_{2}=\cdots=1, b_{1}=b_{2}=-2$ and $\alpha=\beta=0$, the eigenvalues of $A_{n}$ have been found by J. F. Elliott [1] and R. T. Gregory and D. Carney [2] to be

$$
\lambda_{k}=-2+2 \cos \frac{k \pi}{n+1}, \quad k=1, \ldots, n .
$$

When $a_{1}=a_{2}=\cdots=c_{1}=c_{2}=\cdots=1, b_{1}=b_{2}=-2$ and $\alpha=1$ and $\beta=0$ or $\beta=1$, the eigenvalues have been reported to be

$$
\lambda_{k}=-2+2 \cos \frac{k \pi}{n}, \quad k=1, \ldots, n,
$$

and

$$
\lambda_{k}=-2+2 \cos \frac{2 k \pi}{2 n+1}, \quad k=1, \ldots, n,
$$

respectively, without proof.

W. C. Yueh [7] has generalized the results of J. F. Elliott [1] and R. T. Gregory and D. Carney [2] to the case when $a_{1}=a_{2}=\cdots=a$, $c_{1}=c_{2}=\cdots=c, b_{1}=b_{2}=b$ and $\alpha=0, \beta=\sqrt{a c}$ or $\alpha=0, \beta=-\sqrt{a c}$ or $\alpha=-\beta=\sqrt{a c}$ or $\alpha=\beta=\sqrt{a c}$ or $\alpha=\beta=-\sqrt{a c}$. He has calculated, in this case, the eigenvalues and their corresponding eigenvectors

$$
\lambda_{k}=b+2 \sqrt{a c} \cos \theta_{k}, \quad k=1, \ldots, n,
$$

where $\theta_{k}=2 k \pi /(2 n+1),(2 k-1) \pi /(2 n+1),(2 k-1) \pi /(2 n), k \pi / n$ and $(k-1) \pi / n, k=1, \ldots, n$, respectively. 
In [6], we have generalized the results of [7] to more general matrices of the form (1.1) for any complex constants satisfying condition (2) but with $b_{1}=b_{2}$. We have proved that the eigenvalues remain the same as in the case when the $a_{i}$ 's and the $c_{i}$ 's are equal but the components of the eigenvector $u^{(k)}(\sigma)$ associated to the eigenvalue $\lambda_{k}$, which we denote by $u_{j}^{(k)}(\sigma), j=1, \ldots, n$, are of the form

$$
\begin{aligned}
u_{j}^{(k)}(\sigma)= & (-d)^{1-j} a_{\sigma_{1}} \ldots a_{\sigma_{j-1}} u_{1}^{(k)} \\
& \times \frac{d \sin (n-j+1) \theta_{k}-\beta \sin (n-j) \theta_{k}}{d \sin n \theta_{k}-\beta \sin (n-1) \theta_{k}}, \quad j=1, \ldots, n,
\end{aligned}
$$

where $\theta_{k}$ is given by the formula

$$
d^{2} \sin (n+1) \theta_{k}-d(\alpha+\beta) \sin n \theta_{k}+\alpha \beta \sin (n-1) \theta_{k}=0, \quad k=1, \ldots, n .
$$

Recently [4], we generalized the above results to tridiagonal matrices (1.1) satisfying

$$
a_{j} c_{j}=\left\{\begin{array}{ll}
d_{1}^{2} & \text { if } j \text { is odd, } \\
d_{2}^{2} & \text { if } j \text { is even, }
\end{array} \quad j=1,2, \ldots,\right.
$$

where $d_{1}$ and $d_{2}$ are complex numbers, but we always required the diagonal entries to be equal. We have given explicit eigenvalues for many cases, for example when $n$ is even and $\alpha \beta=d_{2}^{2}$, we have

$$
\lambda_{k}= \begin{cases}b+\sqrt{d_{1}^{2}+d_{2}^{2}+2 d_{1} d_{2} \cos \theta_{k}}, & k=1, \ldots, m-1, \\ b-\sqrt{d_{1}^{2}+d_{2}^{2}+2 d_{1} d_{2} \cos \theta_{k}}, & k=m, \ldots, 2 m-2, \\ b+\frac{(\alpha+\beta)+\sqrt{(\alpha-\beta)^{2}+4 d_{1}^{2}}}{2}, & k=n-1, \\ b+\frac{(\alpha+\beta)-\sqrt{(\alpha-\beta)^{2}+4 d_{1}^{2}}}{2}, & k=n .\end{cases}
$$

The corresponding eigenvectors $u^{(k)}(\sigma)=\left(u_{1}^{(k)}(\sigma), \ldots, u_{n}^{(k)}(\sigma)\right)^{t}, k=1, \ldots$, $n-2$, where ${ }^{t}$ is the transposition symbol, are given by

$$
\begin{aligned}
& u_{j}^{(k)}(\sigma) \\
& =\varrho_{j}(\sigma)\left\{\begin{array}{l}
\left(b-\lambda_{k}-\beta\right) \sin \left(\frac{n-j-1}{2}\right) \theta_{k}-\beta \frac{d_{1}}{d_{2}} \sin \left(\frac{n-j-1}{2}\right) \theta_{k} \\
\frac{1}{\sqrt{d_{1} d_{2}}}\left[d_{1} d_{2} \sin \left(\frac{n-j}{2}+1\right) \theta_{k}+\left(d_{2}^{2}-\beta\left(b-\lambda_{k}\right)\right) \sin \frac{n-j}{2} \theta_{k}\right] \\
\text { when } j \text { is even, }
\end{array}\right.
\end{aligned}
$$

where

$$
\varrho_{j}(\sigma)=\left(-\sqrt{d_{1} d_{2}}\right)^{n-j} a_{\sigma_{1}} \ldots a_{\sigma_{j-1}}, \quad j=1, \ldots, n
$$

and 


$$
\theta_{k}= \begin{cases}2 k \pi / n, & k=1, \ldots, m-1, \\ 2(k-m+1) \pi / n, & k=m, \ldots, 2 m-2 .\end{cases}
$$

The eigenvectors $u^{(n-1)}(\sigma)$ and $u^{(n)}(\sigma)$ associated respectively with the eigenvalues $\lambda_{n-1}$ and $\lambda_{n}$ are given by another formula. The matrices studied by J. F. Elliott [1], R. T. Gregory and D. Carney [2] and W. C. Yueh [7] are special cases of those considered by S. Kouachi [6] which are, in their turn, special cases of those considered in this paper since we allow unequal entries on the principal diagonal. All the conditions imposed in the above papers are very restrictive and the techniques used are complicated and are not (in general) applicable to tridiagonal matrices considered in this paper, even for small $n$. For example our techniques are applicable to the $6 \times 6$ matrices

$$
\begin{aligned}
A_{6} & =\left(\begin{array}{cccccc}
5-4 \sqrt{3} & 6 & 0 & 0 & 0 & 0 \\
6 & 3 & 18 & 0 & 0 & 0 \\
0 & 2 & 5 & -4 & 0 & 0 \\
0 & 0 & -9 & 3 & 5+i \sqrt{11} & 0 \\
0 & 0 & 0 & 5-i \sqrt{11} & 5 & -18 i \\
0 & 0 & 0 & 0 & 2 i & 3-3 \sqrt{3}
\end{array}\right), \\
A_{6}^{\prime} & =\left(\begin{array}{cccccc}
5-4 \sqrt{3} & 9 & 0 & 0 & 0 & 0 \\
4 & 3 & 36 & 0 & 0 & 0 \\
0 & 1 & 5 & 12 i & 0 & 0 \\
0 & 0 & -3 i & 3 & 4+2 i \sqrt{5} & 0 \\
0 & 0 & 0 & 4-2 i \sqrt{5} & 5 & -6 \\
0 & 0 & 0 & 0 & -6 & 3-3 \sqrt{3}
\end{array}\right)
\end{aligned}
$$

and guarantee that they possess the same eigenvalues and in addition they give their exact expressions (formulas (9.b) below) since conditions (2) and (3) are satisfied:

$$
\begin{aligned}
\lambda_{1}, \lambda_{4}= & 4 \pm \frac{1}{2} \sqrt{4+16(36) \cos ^{2}\left(\frac{\pi}{6}\right)}=4 \pm \sqrt{109}, \\
\lambda_{2}, \lambda_{5}= & 4 \pm \frac{1}{2} \sqrt{4+16(36) \cos ^{2}\left(\frac{\pi}{3}\right)}=4 \pm \sqrt{37}, \\
\lambda_{3}, \lambda_{6}= & 4-\left(2+\frac{3}{2}\right) \sqrt{3} \\
& \pm \frac{1}{2} \sqrt{(5-3)^{2}+(4 \sqrt{3}+3 \sqrt{3})^{2}-2(5-3)(4 \sqrt{3}-3 \sqrt{3})} \\
= & 4-\left(2+\frac{3}{2}\right) \sqrt{3} \pm \frac{1}{2} \sqrt{151-4 \sqrt{3}},
\end{aligned}
$$


whereas the previous techniques are restricted to the case when the entries on each diagonal are equal and the direct calculation only gives the characteristic polynomial

$$
\begin{aligned}
P(\lambda)= & \lambda^{6}+\lambda^{5}(7 \sqrt{3}-24)+\lambda^{4}(93-139 \sqrt{3}) \\
& +\lambda^{3}(82 \sqrt{3}+1072)+\lambda^{2}(7734 \sqrt{3}-6093) \\
& +\lambda(-10953 \sqrt{3}-1944)-52731 \sqrt{3}+29295
\end{aligned}
$$

whose roots are difficult to calculate.

2. The eigenvalues. When $\alpha=\beta=0$, the matrix $A_{n}(\sigma)$ and its characteristic polynomial will be denoted respectively by $A_{n}^{0}(\sigma)$ and $\Delta_{n}^{0}(\sigma)$, and in the general case they will be denoted by $A_{n}$ and $\Delta_{n}$. We put

$$
Y_{1} Y_{2}=4 d^{2} \cos ^{2} \theta
$$

where

$$
Y_{1}=b_{1}-\lambda \quad \text { and } \quad Y_{2}=b_{2}-\lambda .
$$

The main result of this paper is

TheOREM 1. The eigenvalues of the matrices $A_{n}^{0}(\sigma)$ are independent of the entries $\left(a_{i}, c_{i}, i=1, \ldots, n-1\right)$ and of the mapping $\sigma$ provided that conditions (2) and (3) are satisfied and their characteristic determinants are given by

$$
\begin{array}{ll}
\Delta_{n}^{0}(\sigma)=d^{2 m} \frac{Y_{1} \sin (2 m+2) \theta}{\sin 2 \theta} & \text { when } n=2 m+1, \\
\Delta_{n}^{0}(\sigma)=d^{2 m} \frac{\sin (2 m+1) \theta}{\sin \theta} & \text { when } n=2 m .
\end{array}
$$

Proof. Since the right hand sides of formulas (5.a) and (5.b) are independent of $\sigma$, to prove that so also is $\Delta_{n}^{0}$, it suffices to prove these formulas for $\sigma=$ id. Then, by expanding $\Delta_{n}^{0}$ in terms of its last column and using (2), (3) and (4.1), we get

(6.a.1) $\Delta_{n}^{0}=Y_{1} \Delta_{n-1}^{0}-d^{2} \Delta_{n-2}^{0}, \quad n=3,4, \ldots$, when $n=2 m+1$,

(6.b.1) $\Delta_{n}^{0}=Y_{2} \Delta_{n-1}^{0}-d^{2} \Delta_{n-2}^{0}, \quad n=3,4, \ldots$, when $n=2 m$.

Let us begin by proving (5.b). By writing the expressions of $\Delta_{n}^{0}$ for $n=$ $2 m+2,2 m+1$ and $2 m$, multiplying $\Delta_{2 m+1}^{0}$ and $\Delta_{2 m}^{0}$ by $Y_{2}$ and $d^{2}$ respectively and adding the three resulting equations, we get

$$
\Delta_{2 m+2}^{0}=\left(Y_{1} Y_{2}-2 d^{2}\right) \Delta_{2 m}^{0}-d^{4} \Delta_{2 m-2}^{0} .
$$

We now prove (5.b) by induction on $m$. For $m=0$ and $m=1$ the formula is satisfied. Suppose that it is satisfied for all integers $<m$. Then from (6.b.2) and using (4), we get 


$$
\begin{aligned}
\Delta_{2 m+2}^{0} & =d^{2 m+2}\left[\frac{\left(4 \cos ^{2} \theta-2\right) \sin (2 m+1) \theta-\sin (2 m-1) \theta}{\sin \theta}\right] \\
& =d^{2 m+2}\left[\frac{2 \cos 2 \theta \sin (2 m+1) \theta-\sin (2 m-1) \theta}{\sin \theta}\right] .
\end{aligned}
$$

Using the well known trigonometric formula

$$
2 \sin \eta \cos \zeta=\sin (\eta+\zeta)+\sin (\eta-\zeta)
$$

for $\eta=(2 m+1) \theta$ and $\zeta=2 \theta$, we deduce $(5 . \mathrm{b})$ for $n=2 m+2$.

When $n=2 m+1$, applying (6.b.1) for $n=2 m+2$, we get

$$
\Delta_{2 m+1}^{0}=\frac{\Delta_{2 m+2}^{0}+d^{2} \Delta_{2 m}^{0}}{Y_{2}} .
$$

By direct application of (5.b) twice, for $n=2 m+2$ and $n=2 m$, to the right hand side of the last expression, we get

$$
\Delta_{2 m+1}^{0}=d^{2 m+2} \frac{\sin (2 m+3) \theta+\sin (2 m+1) \theta}{Y_{2} \sin \theta} .
$$

Using again the trigonometric formula $(*)$ for $\eta=(2 m+3) \theta$ and $\zeta=$ $(2 m+1) \theta$, we deduce that

$$
\Delta_{2 m+1}^{0}=2 d^{2 m+2} \frac{\sin (2 m+2) \theta \cos \theta}{Y_{2} \sin \theta} .
$$

By (4), the last expression becomes

$$
\Delta_{2 m+1}^{0}=d^{2 m} \frac{Y_{1} \sin (2 m+2) \theta}{2 \sin \theta \cos \theta},
$$

which gives (5.a) by applying (*) for $\eta=\zeta=\theta$.

THEOREM 2. When conditions (2) and (3) are satisfied, the eigenvalues of $A_{n}^{0}(\sigma)$ are given by

$$
\lambda_{k}= \begin{cases}\frac{\left(b_{1}+b_{2}\right)-\sqrt{\left(b_{1}-b_{2}\right)^{2}+16 d^{2} \cos ^{2} \theta_{k}}}{2}, & k=1, \ldots, m, \\ \frac{\left(b_{1}+b_{2}\right)+\sqrt{\left(b_{1}-b_{2}\right)^{2}+16 d^{2} \cos ^{2} \theta_{k}}}{2}, & k=m+1, \ldots, 2 m, \\ b_{1} & k=n,\end{cases}
$$

where

$$
\begin{aligned}
& \theta_{k}=\left\{\begin{array}{ll}
\frac{k \pi}{2 m+2}, & k=1, \ldots, m, \\
\frac{(k-m) \pi}{2 m+2}, & k=m+1, \ldots, 2 m,
\end{array} \text { when } n=2 m+1,\right.
\end{aligned}
$$


Proof. In formulas (7), the symbol $\sqrt{ }$ denotes any concrete branch of the square root. When $n=2 m+1$, the eigenvalues are trivial consequences of formula (5.a) by putting $Y_{1} \sin (2 m+2) \theta=0$, which gives, by using (4) and (4.1),

$$
\lambda^{2}-\left(b_{1}+b_{2}\right) \lambda+b_{1} b_{2}-4 d^{2} \cos ^{2} \theta_{k}=0, \quad k=1, \ldots, m,
$$

where $\theta_{k}=k \pi /(2 m+2), k=1, \ldots, m$ and $\lambda_{n}=b_{1}$.

When $n=2 m$, the same reasoning with $\sin (2 m+1) \theta=0$ yields $(7)$.

If we suppose that $\alpha \neq 0$ or $\beta \neq 0$, then following the same reasoning as in S. Kouachi [4] and [5] by expanding $\Delta_{n}$ in terms of the first and last columns and using the linearity of determinants with respect to their columns, we get

$$
\Delta_{n}=\Delta_{n}^{0}-\alpha\left|E_{n-1}^{2}\right|-\beta\left|E_{n-1}^{1}\right|+\alpha \beta\left|\begin{array}{ccccc}
Y_{2} & c_{2} & 0 & \ldots & 0 \\
a_{2} & Y_{3} & \ddots & \ddots & \vdots \\
0 & \ddots & \ddots & \ddots & 0 \\
\vdots & \ddots & \ddots & \ddots & c_{n-2} \\
0 & \ldots & 0 & a_{n-2} & Y_{n-1}
\end{array}\right|,
$$

where $E_{n-1}^{1}$ and $E_{n-1}^{2}$ are $(n-1) \times(n-1)$ matrices of the form (1),

$$
E_{n-1}^{i}=\left(\begin{array}{ccccc}
Y_{i} & c_{i} & 0 & \ldots & 0 \\
a_{i} & Y_{i+1} & \ddots & \ddots & \vdots \\
0 & \ddots & \ddots & \ddots & 0 \\
\vdots & \ddots & \ddots & \ddots & c_{n+i-3} \\
\ldots & \ldots & 0 & a_{n+i-3} & Y_{n+i-2}
\end{array}\right), \quad i=1,2 .
$$

Since all the entries $a_{i}$ on the subdiagonal and $c_{i}$ on the superdiagonal satisfy conditions (2) and (3), using formulas (5.a) and (5.b) and taking into account the order of the entries $a_{i}, c_{i}$ and $Y_{i}$, we deduce

$$
\begin{aligned}
\Delta_{n}= & d^{2 m} \frac{Y_{1} \sin (2 m+2) \theta}{\sin 2 \theta}-(\alpha+\beta) d^{2 m} \frac{\sin (2 m+1) \theta}{\sin \theta} \\
& +\alpha \beta d^{2 m-2} \frac{Y_{2} \sin 2 m \theta}{\sin 2 \theta} \\
= & d^{2 m} \frac{Y_{1} \sin (2 m+2) \theta-2(\alpha+\beta) \cos \theta \sin (2 m+1) \theta+\frac{\alpha \beta}{d^{2}} Y_{2} \sin 2 m \theta}{\sin 2 \theta} .
\end{aligned}
$$

Applying the trigonometric formula $(*)$ for $\eta=(2 m+1) \theta$ and $\zeta=\theta$, we get

$$
\Delta_{n}=d^{2 m} \frac{\left[Y_{1}-(\alpha+\beta)\right] \sin (2 m+2) \theta+\left[\frac{\alpha \beta}{d^{2}} Y_{2}-(\alpha+\beta)\right] \sin 2 m \theta}{\sin 2 \theta}
$$


when $n=2 m+1$, and

$$
\begin{aligned}
& \Delta_{n}=d^{2 m} \frac{\sin (2 m+1) \theta}{\sin \theta}-\alpha d^{2 m-2} \frac{Y_{2} \sin 2 m \theta}{\sin 2 \theta} \\
& \quad-\beta d^{2 m-2} \frac{Y_{1} \sin 2 m \theta}{\sin 2 \theta}+\alpha \beta d^{2 m-2} \frac{\sin (2 m-1) \theta}{\sin \theta} \\
& =d^{2 m-2} \frac{2 d^{2} \sin (2 m+1) \theta \cos \theta-\left(\alpha Y_{2}+\beta Y_{1}\right) \sin 2 m \theta+2 \alpha \beta \sin (2 m-1) \theta \cos \theta}{\sin 2 \theta}
\end{aligned}
$$

when $n=2 m$. Applying $(*)$ twice, first for $\eta=(2 m+1) \theta$ and $\zeta=\theta$ and then for $=(2 m-1) \theta$ and $\zeta=\theta$, we get

(8.b) $\Delta_{n}=$

$$
d^{2 m-2} \frac{d^{2} \sin (2 m+2) \theta-\left(\alpha Y_{2}+\beta Y_{1}-\alpha \beta-d^{2}\right) \sin 2 m \theta+\alpha \beta \sin (2 m-2) \theta}{\sin 2 \theta} .
$$

If $\alpha \beta=d^{2}$, then by application of $(*)$ for $\eta=2 m \theta$ and $\zeta=2 \theta$, we get

$$
\Delta_{n}=d^{2 m-2} \frac{\left(2 d^{2} \cos 2 \theta-\alpha Y_{2}-\beta Y_{1}+2 d^{2}\right) \sin 2 m \theta}{\sin 2 \theta} .
$$

Using (4) and the formula $\cos 2 \theta=2 \cos ^{2} \theta-1$, we deduce

$$
\Delta_{n}=d^{2 m-2} \frac{\left(Y_{1} Y_{2}-\alpha Y_{2}-\beta Y_{1}\right) \sin 2 m \theta}{\sin 2 \theta} .
$$

Before proceeding further, let us deduce from formulas (8) a proposition for the matrix $B_{n}(\sigma)$ which is obtained from $A_{n}(\sigma)$ by changing $\alpha$ and $\beta$ to $b_{1}$ and $b_{2}$ respectively.

Proposition 1. When $n$ is even, the eigenvalues of $B_{n}(\sigma)$ are the same as those of $A_{n}(\sigma)$.

We have

THEOREM 3. When $n=2 m$ and $\alpha \beta=d^{2}$, if conditions (2) and (3) are satisfied, then the eigenvalues of all the matrices $A_{n}(\sigma)$ are given by

(9.b) $\quad \lambda_{k}=$

$$
\left\{\begin{array}{l}
\frac{\left(b_{1}+b_{2}\right)-\sqrt{\left(b_{1}-b_{2}\right)^{2}+16 d^{2} \cos ^{2} \theta_{k}}}{2}, k=1, \ldots, m-1, \\
\frac{\left(b_{1}+b_{2}\right)+\sqrt{\left(b_{1}-b_{2}\right)^{2}+16 d^{2} \cos ^{2} \theta_{k}}}{2}, k=m, \ldots, 2 m-2, \\
\frac{\left(b_{1}+b_{2}-\alpha-\beta\right)-\sqrt{\left(b_{1}+b_{2}\right)^{2}+(\alpha+\beta)^{2}-2\left(b_{1}-b_{2}\right)(\alpha-\beta)}}{2}, k=n-1, \\
\frac{\left(b_{1}+b_{2}-\alpha-\beta\right)+\sqrt{\left(b_{1}+b_{2}\right)^{2}+(\alpha+\beta)^{2}-2\left(b_{1}-b_{2}\right)(\alpha-\beta)}}{2}, k=n,
\end{array}\right.
$$


where

$$
\theta_{k}= \begin{cases}\frac{k \pi}{2 m}, & k=1, \ldots, m-1, \\ \frac{(k-m+1) \pi}{2 m}, & k=m, \ldots, 2 m-2 .\end{cases}
$$

Proof. By (4.1), the characteristic determinant (8.b.1) becomes

$$
\Delta_{n}=d^{2 m-2} \frac{\left[\lambda^{2}-\left(b_{1}+b_{2}-\alpha-\beta\right) \lambda-\left(\alpha b_{2}+\beta b_{1}-b_{1} b_{2}\right)\right] \sin 2 m \theta}{\sin 2 \theta} .
$$

Then the eigenvalues $\lambda_{k}, k=1, \ldots, 2 m-2$, are trivial by putting $2 m \theta=$ $k \pi, k=1, \ldots, m-1$, and using (4). This gives

$$
\lambda^{2}-\left(b_{1}+b_{2}\right) \lambda+b_{1} b_{2}-4 d^{2} \cos ^{2} \theta_{k}=0, \quad k=1, \ldots, m-1 .
$$

The eigenvalues $\lambda_{n-1}$ and $\lambda_{n}$ are deduced by solving the equation

$$
\lambda^{2}-\left(b_{1}+b_{2}-\alpha-\beta\right) \lambda-\left(\alpha b_{2}+\beta b_{1}-b_{1} b_{2}\right)=0 .
$$

Let us see what formulas (8) say and what they do not say. They say that if $a_{i}^{\prime}, c_{i}^{\prime}, i=1, \ldots, n-1$, are other constants satisfying conditions (2) and (3) and

$$
A_{n}^{\prime}=\left(\begin{array}{cccccc}
-\alpha+b_{1} & c_{1}^{\prime} & 0 & 0 & \ldots & 0 \\
a_{1}^{\prime} & b_{2} & c_{2}^{\prime} & 0 & \ldots & 0 \\
0 & a_{2}^{\prime} & b_{3} & \ddots & \ddots & \vdots \\
0 & 0 & \ddots & \ddots & \ddots & 0 \\
\vdots & \vdots & \ddots & \ddots & \ddots & c_{n-1}^{\prime} \\
0 & \ldots & \ldots & 0 & a_{n-1}^{\prime} & -\beta+b_{n}
\end{array}\right),
$$

then the matrices $A_{n}, A_{n}^{\prime}$ and $A_{n}(\sigma)$ possess the same characteristic polynomial and hence the same eigenvalues. Therefore we have this immediate consequence of formulas (4):

COROLlary 1. The matrices $A_{n}, A_{n}^{\prime}$ and $A_{n}(\sigma)$ are all similar provided that all the entries satisfy conditions (2) and (3).

3. The eigenvectors. The components of the eigenvector $u^{(k)}(\sigma), k=$ $1, \ldots, n$, associated to the eigenvalue $\lambda_{k}, k=1, \ldots, n$, which we denote by $u_{j}^{(k)}, j=1, \ldots, n$, are solutions of the linear system of equations

$$
\left\{\begin{array}{c}
\left(-\alpha+\xi_{1}^{(k)}\right) u_{1}^{(k)}+c_{\sigma_{1}} u_{2}^{(k)}=0 \\
a_{\sigma_{1}} u_{1}^{(k)}+\xi_{2}^{(k)} u_{2}^{(k)}+c_{\sigma_{2}} u_{3}^{(k)}=0 \\
\ldots \ldots \ldots \ldots \ldots \ldots \ldots \ldots \ldots \ldots \ldots \\
a_{\sigma_{n-1}} u_{n-1}^{(k)}+\left(-\beta+\xi_{n}^{(k)}\right) u_{n}^{(k)}=0
\end{array}\right.
$$


where

$$
\begin{aligned}
\xi_{i}^{(k)} & =Y_{i}^{(k)}=b_{i}-\lambda_{k} \\
& =\left\{\begin{array}{ll}
b_{1}-\lambda_{k} & \text { if } i \text { is odd, } \\
b_{2}-\lambda_{k} & \text { if } i \text { is even, }
\end{array} \quad i=1, \ldots, n-1, k=1, \ldots, n,\right.
\end{aligned}
$$

given by formulas (4), and $\theta_{k}, k=1, \ldots, n$, are solutions of

$$
\text { (11.a) }\left[\xi_{1}^{(k)}-(\alpha+\beta)\right] \sin (2 m+2) \theta_{k}+\left[\frac{\alpha \beta}{d^{2}} \xi_{2}^{(k)}-(\alpha+\beta)\right] \sin 2 m \theta_{k}=0
$$

when $n=2 m+1$, and

$$
\begin{aligned}
d^{2} \sin (2 m+2) \theta_{k}-\left(\alpha \xi_{2}^{(k)}+\beta \xi_{1}^{(k)}-\alpha \beta\right. & \left.-d^{2}\right) \sin 2 m \theta_{k} \\
& +\alpha \beta \sin (2 m-2) \theta_{k}=0
\end{aligned}
$$

when $n=2 m$.

Since these $n$ equations are linearly dependent, by eliminating the first equation we obtain a system of $n-1$ equations in $n-1$ unknowns, written in matrix form as

$$
\left(\begin{array}{ccccc}
\xi_{2}^{(k)} & c_{\sigma_{2}} & 0 & \ldots & 0 \\
a_{\sigma_{2}} & \xi_{3}^{(k)} & \ddots & \ddots & \vdots \\
0 & \ddots & \ddots & \ddots & 0 \\
\vdots & \ddots & \ddots & \ddots & c_{\sigma_{n-1}} \\
0 & \ldots & 0 & a_{\sigma_{n-1}} & -\beta+\xi_{n}^{(k)}
\end{array}\right)\left(\begin{array}{c}
u_{2}^{(k)} \\
u_{3}^{(k)} \\
\vdots \\
\vdots \\
u_{n}^{(k)}
\end{array}\right)=\left(\begin{array}{c}
-a_{\sigma_{1}} u_{1}^{(k)} \\
0 \\
\vdots \\
\vdots \\
0
\end{array}\right)
$$

The determinant of this system is given by formulas (8) for $\alpha=0$ and $n$ replaced by $n-1$ and equals

$$
\Delta_{n-1}^{(k)}=d^{2 m-2} \frac{d^{2} \sin (2 m+2) \theta_{k}-\left(\beta \xi_{2}^{(k)}-d^{2}\right) \sin 2 m \theta_{k}}{\sin 2 \theta_{k}}
$$

when $n=2 m+1$, and

$$
\Delta_{n-1}^{(k)}=d^{2 m-2} \frac{\left(\xi_{2}^{(k)}-\beta\right) \sin 2 m \theta_{k}-\beta \sin (2 m-2) \theta_{k}}{\sin 2 \theta_{k}}
$$

when $n=2 m$, and for all $k=1, \ldots, n$.

$$
u_{j}^{(k)}(\sigma)=\frac{\Gamma_{j}^{(k)}(\sigma)}{\Delta_{n-1}^{(k)}}, \quad j, k=1, \ldots, n,
$$

where 
$\Gamma_{j}^{(k)}(\sigma)$

$$
=\left|\begin{array}{cccccccc}
\xi_{2}^{(k)} & c_{\sigma_{2}} & 0 & \ldots & -a_{\sigma_{1}} u_{1}^{(k)} & 0 & \ldots & 0 \\
a_{\sigma_{2}} & \xi_{3}^{(k)} & \ddots & \ddots & 0 & 0 & \ldots & \vdots \\
0 & \ddots & \ddots & c_{\sigma_{j-2}} & \vdots & \vdots & \ldots & \vdots \\
0 & 0 & a_{\sigma_{j-2}} & \xi_{j-1}^{(k)} & 0 & 0 & \ldots & \vdots \\
\vdots & \vdots & \ddots & a_{\sigma_{j-1}} & 0 & c_{\sigma_{j}} & \ddots & \vdots \\
\vdots & \vdots & \ddots & \ddots & 0 & \xi_{j+1}^{(k)} & \ddots & 0 \\
\vdots & \vdots & \ldots & \ddots & \vdots & a_{\sigma_{j}+1} \cdot & \ddots & c_{\sigma_{n-1}} \\
0 & \ldots & \ldots & \ldots & 0 & 0 & a_{\sigma_{n-1}} & -\beta+\xi_{n}^{(k)}
\end{array}\right|,
$$

$j=2, \ldots, n, k=1, \ldots, n$. By permuting the first $j-2$ columns with the $(j-1)$ th one and using the properties of determinants, we get

$$
u_{j}^{(k)}(\sigma)=(-1)^{j-2} \frac{\Lambda_{j}^{(k)}(\sigma)}{\Delta_{n-1}}, \quad j=2, \ldots, n,
$$

where $\Lambda_{j}^{(k)}(\sigma)$ is the determinant of the matrix

where

$$
C_{j}^{(k)}(\sigma)=\left(\begin{array}{cc}
T_{j-1}^{(k)}(\sigma) & \mathbf{0} \\
\mathbf{0} & S_{n-j}^{(k)}(\sigma)
\end{array}\right)
$$

$$
T_{j-1}^{(k)}(\sigma)=\left(\begin{array}{cccccc}
-a_{\sigma_{1}} u_{1}^{(k)} & \xi_{2}^{(k)} & c_{\sigma_{2}} & 0 & \cdots & 0 \\
0 & a_{\sigma_{2}} & \ddots & \ddots & \ddots & \vdots \\
\vdots & \ddots & \ddots & \ddots & \ddots & 0 \\
\vdots & 0 & \ddots & \ddots & \ddots & c_{\sigma_{j-2}} \\
\vdots & 0 & 0 & \ddots & \ddots & \xi_{j-1}^{(k)} \\
0 & \cdots & \cdots & \cdots & 0 & a_{\sigma_{j-1}}
\end{array}\right)
$$

is a supertriangular $(j-1) \times(j-1)$ matrix with diagonal $\left(-a_{\sigma_{1}} u_{1}^{(k)}, a_{\sigma_{2}}, \ldots\right.$, $\left.a_{\sigma_{j-1}}\right)$ and

$$
S_{n-j}^{(k)}(\sigma)=\left(\begin{array}{ccccc}
\xi_{j+1}^{(k)} & c_{\sigma_{j+1}} & 0 & \cdots & 0 \\
a_{\sigma_{j}+1} & \ddots & \ddots & \ddots & \vdots \\
0 & \ddots & \ddots & \ddots & 0 \\
\vdots & \ddots & \ddots & \ddots & c_{\sigma_{n-1}} \\
0 & \ldots & 0 & a_{\sigma_{n-1}} & -\beta+\xi_{n}^{(k)}
\end{array}\right),
$$


is an $(n-j) \times(n-j)$ tridiagonal matrix of the form (1.1) and satisfying conditions (2) and (3). Thus

$$
\begin{aligned}
\left|C_{j}^{(k)}(\sigma)\right| & =\left|T_{j-1}^{(k)}(\sigma)\right|\left|S_{n-j}^{(k)}(\sigma)\right| \\
& =-a_{\sigma_{1}} \ldots a_{\sigma_{j-1}} u_{1}^{(k)} \Delta_{n-j}^{(k)}, \quad j=2, \ldots, n \text { and } k=1, \ldots, n
\end{aligned}
$$

where $\Delta_{n-j}^{(k)}(\sigma)$ is given by formulas (8) for $\alpha=0$ and $n$ replaced by $n-j$ :

$$
\Delta_{n-j}^{(k)}= \begin{cases}d^{n-j-2} \frac{d^{2} \sin (n-j+2) \theta_{k}-\left(\beta \xi_{2}^{(k)}-d^{2}\right) \sin (n-j) \theta_{k}}{\sin 2 \theta_{k}} & \text { for } j \text { odd, } \\ d^{n-j-1} \frac{\left[\xi_{1}^{(k)}-\beta\right] \sin (n-j+1) \theta_{k}-\beta \sin (n-j-1) \theta_{k}}{\sin 2 \theta_{k}} & \text { for } j \text { even, }\end{cases}
$$

when $n$ is odd, and

$$
\Delta_{n-j}^{(k)}= \begin{cases}d^{n-j-1} \frac{\left[\xi_{2}^{(k)}-\beta\right] \sin (n-j+1) \theta_{k}-\beta \sin (n-j-1) \theta_{k}}{\sin 2 \theta_{k}} & \text { for } j \text { odd } \\ d^{n-j-2} \frac{d^{2} \sin (n-j+2) \theta_{k}-\left(\beta \xi_{1}^{(k)}-d^{2}\right) \sin (n-j) \theta_{k}}{\sin 2 \theta_{k}} & \text { for } j \text { even, }\end{cases}
$$

when $n$ is even, for all $j=2, \ldots, n$ and $k=1, \ldots, n$. Using formulas (13)(17), we get

(18) $u_{j}^{(k)}(\sigma)=(-1)^{j-1} a_{\sigma_{1}} \ldots a_{\sigma_{j-1}} u_{1}^{(k)} \frac{\Delta_{n-j}^{(k)}}{\Delta_{n-1}^{(k)}}, \quad j=2, \ldots, n, k=1, \ldots, n$.

Finally,

$$
\begin{aligned}
& u_{j}^{(k)}(\sigma)= \\
& \mu_{j}(\sigma) u_{1}^{(k)} \begin{cases}d^{1-j} \frac{d^{2} \sin (n-j+2) \theta_{k}-\left(\beta \xi_{2}^{(k)}-d^{2}\right) \sin (n-j) \theta_{k}}{d^{2} \sin (n+1) \theta_{k}-\left(\beta \xi_{2}^{(k)}-d^{2}\right) \sin (n-1) \theta_{k}} & \text { for } j \text { odd, } \\
d^{2-j} \frac{\left(\xi_{1}^{(k)}-\beta\right) \sin (n-j+1) \theta_{k}-\beta \sin (n-j-1) \theta_{k}}{d^{2} \sin (n+1) \theta_{k}-\left(\beta \xi_{2}^{(k)}-d^{2}\right) \sin (n-1) \theta_{k}} & \text { for } j \text { even, }\end{cases}
\end{aligned}
$$

when $n$ is odd, and

$$
\begin{aligned}
& u_{j}^{(k)}(\sigma)= \\
& \mu_{j}(\sigma) u_{1}^{(k)} \begin{cases}d^{1-j} \frac{\left(\xi_{2}^{(k)}-\beta\right) \sin (n-j+1) \theta_{k}-\beta \sin (n-j-1) \theta_{k}}{\left(\xi_{2}^{(k)}-\beta\right) \sin n \theta_{k}-\beta \sin (n-2) \theta_{k}} & \text { for } j \text { odd, } \\
d^{-j} \frac{d^{2} \sin (n-j+2) \theta_{k}-\left(\beta \xi_{1}^{(k)}-d^{2}\right) \sin (n-j) \theta_{k}}{\left(\xi_{2}^{(k)}-\beta\right) \sin n \theta_{k}-\beta \sin (n-2) \theta_{k}} & \text { for } j \text { even, }\end{cases}
\end{aligned}
$$

for all $j=2, \ldots, n$ and $k=1, \ldots, n$, when $n$ is even, where

$$
\mu_{j}(\sigma)=(-1)^{1-j} a_{\sigma_{1}} \ldots a_{\sigma_{j-1}}, \quad j=2, \ldots, n,
$$

$\xi_{1}^{(k)}, \xi_{2}^{(k)}$ and $\theta_{k}, k=1, \ldots, n$, are given by formulas (4) and (11). If we choose 
$u_{1}^{(k)}(\sigma)=(-d)^{n-1} \begin{cases}d^{2} \sin (n+1) \theta_{k}-\left(\beta \xi_{2}^{(k)}-d^{2}\right) \sin (n-1) \theta_{k} & \text { for } n \text { odd, } \\ \left(\xi_{2}^{(k)}-\beta\right) \sin n \theta_{k}-\beta \sin (n-2) \theta_{k} & \text { for } n \text { even, }\end{cases}$

and put

$$
\varrho_{j}(\sigma)=(-d)^{n-j} a_{\sigma_{1}} \ldots a_{\sigma_{j-1}}, \quad j=2, \ldots, n
$$

we get

TheOREM 4. The eigenvectors $u^{(k)}(\sigma)=\left(u_{1}^{(k)}(\sigma), \ldots, u_{n}^{(k)}(\sigma)\right)^{t}, k=$ $1, \ldots, n$ of the matrices $A_{n}(\sigma)$ are given by

(19.a) $u_{j}^{(k)}(\sigma)=\varrho_{j}(\sigma)\left\{\begin{array}{r}d^{2} \sin (n-j+2) \theta_{k}-\left(\beta \xi_{2}^{(k)}-d^{2}\right) \sin (n-j) \theta_{k} \\ \text { for } j \text { odd }, \\ \left(\xi_{1}^{(k)}-\beta\right) d \sin (n-j+1) \theta_{k}-\beta d \sin (n-j-1) \theta_{k} \\ \text { for } j \text { even, }\end{array}\right.$ when $n$ is odd, and

$$
u_{j}^{(k)}(\sigma)=\varrho_{j}(\sigma)\left\{\begin{array}{r}
\left(\xi_{2}^{(k)}-\beta\right) \sin (n-j+1) \theta_{k}-\beta \sin (n-j-1) \theta_{k} \\
\quad \text { for } j \text { odd }, \\
d \sin (n-j+2) \theta_{k}-\left(\beta \xi_{1}^{(k)} / d-d\right) \sin (n-j) \theta_{k} \\
\text { for } j \text { even },
\end{array}\right.
$$

when $n$ is even. Here $\varrho_{j}(\sigma), j=2, \ldots, n$, is given by $(\ddagger)$ and $\theta_{k}, \xi_{1}^{(k)}$ and $\xi_{2}^{(k)}, k=1, \ldots, n$, are given by formulas (4) and (11).

Acknowledgements. The author thanks the Superior Education Ministry of Algeria and the National Agency of Development and Scientific Research (ANDRU) for their financial support. He also thanks the anonymous referees for their suggestions.

\section{References}

[1] J. F. Elliott, The characteristic roots of certain real symmetric matrices, master thesis, Univ. of Tennessee, 1953.

[2] R. T. Gregory and D. Carney, A Collection of Matrices for Testing Computational Algorithms, Wiley-Interscience, 1969.

[3] U. Grenander and G. Szegö, Toeplitz Forms and Their Applications, Univ. of California Press, Berkeley and Los Angeles, 1958.

[4] S. Kouachi, Eigenvalues and eigenvectors of tridiagonal matrices, Electronic J. Linear Algebra 15 (2006), 115-133.

[5] - Explicit eigenvalues of tridiagonal matrices, in preparation.

[6] - Eigenvalues and eigenvectors of several tridiagonal matrices, submitted to Bull. Belgian Math. Soc. 
[7] W. C. Yueh, Eigenvalues of several tridiagonal matrices, Appl. Math. E-Notes 5 (2005), 66-74.

Centre Universitaire de Khenchela

40100 Algeria

E-mail: kouachi@hotmail.com

Current address:

Laboratoire de Mathématiques

Université d'Annaba

23200 Algeria 